\title{
Purulent Necrotic Dislocation of the Hip Joint Associated with Umbilical Infection in a Foal
}

\author{
Fumie MAGATA $^{1}$, Mitsuo ISHII ${ }^{1}$, Eri OIKAWA ${ }^{2}$, Hidefumi FURUOKA ${ }^{2}$, Kazutaka YAMADA ${ }^{1}$, \\ Naoki SASAKI ${ }^{1}$, Syo SHIMIZU $^{3}$ and Hisashi INOKUMA ${ }^{1}$ \\ ${ }^{1}$ Department of Applied Veterinary Medicine, ${ }^{2}$ Department of Basic Veterinary Medicine, Obihiro University \\ of Agriculture and Veterinary Medicine, 11 Nishi-2-Sen, Inada-cho, Obihiro, Hokkaido 080-8555, ${ }^{3}$ Eastern \\ Clinical Center of Tokachi Agricultural Mutual Aid Association, 59 Kisen, Kawanishi-cho, Obihiro, Hokkaido \\ 089-1182, Japan
}

\begin{abstract}
A 42-day-old heavy draft horse fell into sudden astasia. Significant swelling and heat sensation of the left femoral region were observed. Because of a friction sound in the left hip, we supposed that the hip joint was dislocated or the hip bone was fractured. Computed Tomography (CT) examination showed that the left hip joint was dislocated and the left femoral head was disjunct. We carried out a pathological autopsy, and made a diagnosis of the foal as fracture of the hip bone and femoral head with suppurative umbilical arteritis. Pathologic changes in the umbilical artery and hind leg were completely unilateral, suggesting that left umbilical arteritis spread to the blood circulation, causing arthritis and dislocation of the hip bone.
\end{abstract}

Key words: dislocation, foal, umbilical infection

Infection of umbilical cord remnants in foals develops during the neonatal period [2]. A dirty environment at the time of birth and poor hygiene of the umbilicus predisposes foals to these infections [1]. Umbilical infections may involve one or more of the internal structures, although the urachus and arteries are most commonly infected [2]. They present symptoms such as swelling of the umbilical region, heat sensation and flare, but often exist without any external evidence $[1,6,8]$. Sometimes, umbilical infections are accompanied with septicemia or other localized infections such as pneumonia, diarrhea and arthritis caused by hematogenous spread [1]. In the present report, we describe a case of coxarthritis and dislocation of the hip joint due to umbilical infection.

A 42-day-old heavy draft horse (mare, bred in Tokachi, Hokkaido district) fell into sudden astasia without any abnormality after birth. The foal had a high rectal temperature $\left(40.0^{\circ} \mathrm{C}\right)$, and the left thigh of the foal was swollen at the first medical examination.

This article was accepted March 23, 2010

*Corresponding author. e-mail: polkadot.jemini@gmail.com
J. Equine Sci.

Vol. 21, No. 2

pp. 17-20, 2010
The foal was treated with antibiotics, fluid infusion, and anti-inflammatory agents. After 15 days treatment, astasia or dysstasia was still present, and the swelling of the left thigh did not improve. The foal was taken to the Obihiro University of Agriculture and Veterinary Medicine on the 57 th day after birth.

At the first examination, swelling and heat sensation of the upper thigh were observed. Because of a friction sound in the left hip during passive movement, we supposed that the hip joint was dislocated or the bone was fractured. The growth status and condition of the nutrition were normal. Hematological examination showed leukocytosis $(13,300 / \mu l)$. Serum biochemical analysis showed a low level of albumin $(1.82 \mathrm{~g} / \mathrm{d} l)$ and elevation of $\alpha(1.79 \mathrm{~g} / \mathrm{d} l), \beta(2.76 \mathrm{~g} / \mathrm{d} l)$, and $\gamma(1.02 \mathrm{~g} /$ $\mathrm{d} l$ ) globulin, therefore the albumin/globulin ratio was greatly decreased $(0.33)$ in the serum protein fraction (Table 1).

X-ray examination of the pelvic part revealed that the outline of the femoral head was indistinct in the ventral-dorsal direction. A CT examination was carried out under sedation using a multi-detector row CT (Asteion Super 4, Toshiba, Tokyo, Japan). The CT 
Table 1. Hematological examination and serum biochemical analysis

\begin{tabular}{llll}
\hline $\mathrm{RBC}$ & $9.37 \times 10^{6} / \mu l$ & $\mathrm{GOT}$ & $179 \mathrm{IU} / l$ \\
$\mathrm{Hb}$ & $9.4 \mathrm{~g} / \mathrm{d} l$ & ALP & $389 \mathrm{IU} / l$ \\
$\mathrm{PCV}$ & $28.6 \%$ & $\mathrm{LDH}$ & $434 \mathrm{IU} / l$ \\
$\mathrm{MCV}$ & $30.5 \mathrm{f} l$ & $\gamma-\mathrm{GTP}$ & $19 \mathrm{IU} / l$ \\
$\mathrm{MCH}$ & $10.1 \mathrm{pg}$ & $\mathrm{CPK}$ & $163 \mathrm{IU} / l$ \\
$\mathrm{MCHC}$ & $32.9 \%$ & $\mathrm{BUN}$ & $16.2 \mathrm{mg} / \mathrm{d} l$ \\
Platelet & $31.6 \times 10^{4} / \mu l$ & $\mathrm{Cre}$ & $0.8 \mathrm{mg} / \mathrm{d} l$ \\
WBC & $13,300 / \mu l$ & $\mathrm{Na}$ & $133 \mathrm{mEq} / l$ \\
$\mathrm{TP}$ & $7.4 \mathrm{~g} / \mathrm{d} l$ & $\mathrm{~K}$ & $2.8 \mathrm{mEq} / l$ \\
Alb & $1.82 \mathrm{~g} / \mathrm{d} l(24.6 \%)$ & $\mathrm{Cl}$ & $100 \mathrm{mEq} / l$ \\
$\alpha-\mathrm{Glb}$ & $1.79 \mathrm{~g} / \mathrm{d} l(24.3 \%)$ & $\mathrm{Ca}$ & $9.7 \mathrm{mg} / \mathrm{d} l$ \\
$\beta$-Glb & $2.76 \mathrm{~g} / \mathrm{d} l(37.3 \%)$ & $\mathrm{P}$ & $4.9 \mathrm{mg} / \mathrm{d} l$ \\
$\gamma-\mathrm{Glb}$ & $1.02 \mathrm{~g} / \mathrm{d} l(13.8 \%)$ & $\mathrm{Mg}$ & $1.2 \mathrm{mg} / \mathrm{d} l$ \\
$\mathrm{~A} / \mathrm{G}$ & 0.33 & & \\
\hline
\end{tabular}

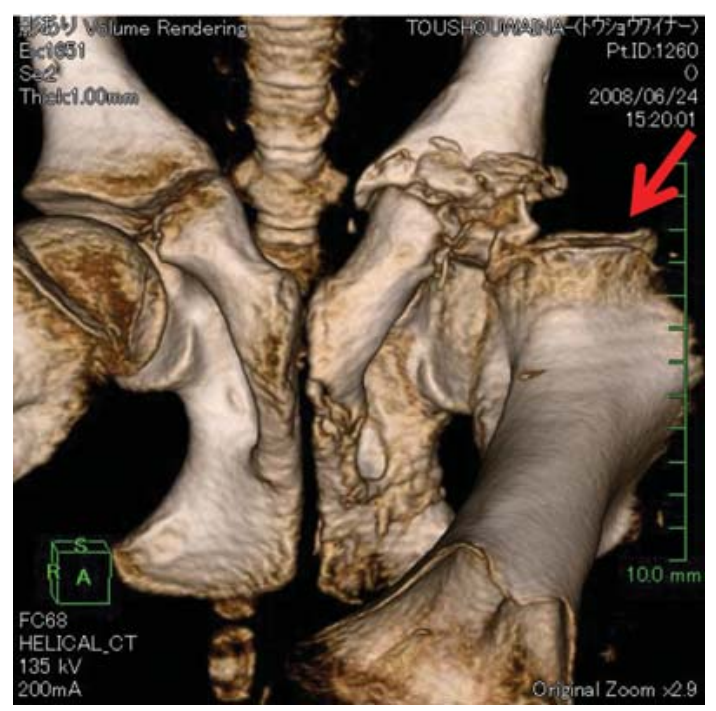

Fig. 1. CT examination of the hip joint and thigh showed disjunction of the left femoral head and dislocation of the left hip joint (arrow).

examination showed dislocation of the left hip joint in the craniodorsal region. A low absorption region (10 Hounsfield units), including a gas image, was shown in the left femoral head surroundings, suggesting the existence of an abscess. After CT imaging, 3D volume renderings were created immediately in Digital Imaging and Communications in Medicine (DICOM) format using a dedicated image processing workstation (Virtual Place Advance PLUS, AZE, Tokyo, Japan). The 3D image showed disjunction of the left femoral head and a bone fracture of the left pelvic symphysis (pubic bones) (Fig. 1). Because of poor prognosis, the foal was euthanized and a pathological autopsy was

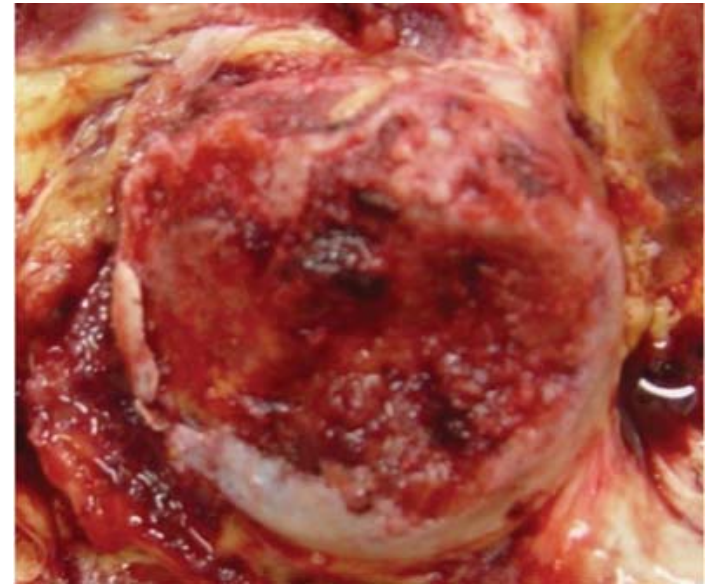

Fig. 2. The left femoral head was about half the normal size and showed osteonecrosis and osteolysis. The ligament of the femoral head was necrotic with hemorrhage.

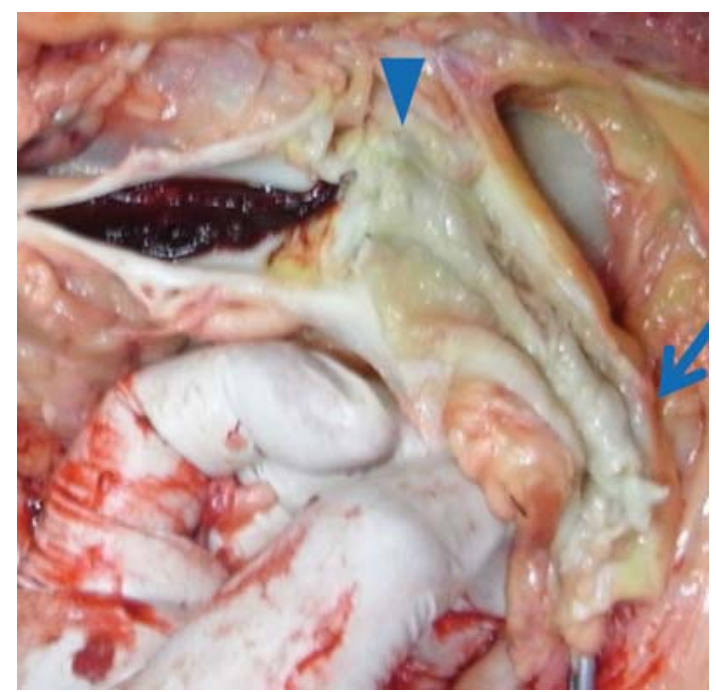

Fig. 3. Left umbilical artery filled with yellowish-white thrombus (arrow). The thrombus spread to the point of divergence from the internal pudendal artery (arrow head).

carried out on the 58th day after birth.

In the left hip joint, the left femoral head (acetabular side) was about half the normal size due to osteonecrosis and osteolysis. The ligament of the femoral head was necrotic with hemorrhage (Fig. 2). Moreover, the hip bone was separated into three parts in the acetabulum, and osteomyelitis with a small abscess was observed in the pubis. There were some abscesses and suppurative inflammation in wide areas 
of the left femoral region and hind leg. Muscles of the hind leg showed massive necrosis with subcutaneous edema. The wall of the left umbilical artery was thick, and the umbilical artery was filled with a yellowish-white purulent thrombus measuring $15 \mathrm{~cm}$ in length. The thrombus of the umbilical artery spread to the point of divergence from the internal pudendal artery (Fig. 3). A white thrombus was formed in the left femoral vein. Histopathologically, the left umbilical artery showed purulent vasculitis with thrombus. Macrophages, neutrophils and lymphocytes infiltrated into the wall of the artery. In the spleen and some lymph nodes, atrophy of lymphoid follicles was observed. In a bacteriological examination, no bacteria were detected the area of hip joint necrosis, the pubic marrow and the purulent thrombus in the umbilical artery. However, a Gram-positive short bacillus was detected from the abscess in the lower thigh.

On the basis of these findings, we made a diagnosis of fracture of the hip bone and femoral head with suppurative umbilical arteritis.

In the present case, suppurative inflammation was observed in the left umbilical artery. In addition, some abscesses and inflammation of muscles were found in wide areas of the left femoral region and hind leg, but were not seen in the right umbilical artery and the right hind leg. These results suggest that bacterial infection of the umbilical cord caused left umbilical arteritis, and the infection spread to the blood circulation, causing the disease of the left hind leg. After birth, foals develop their immune system through intake of colostrum [4, 7]. But if the immune system fails to develop, septicemia may occur causing various systemic symptoms and arthritis of limbs [1,2]. We did not carry out an immunologic examination of the foal, so we could not evaluate whether the foal's immune system had successfully developed or not. However, the foal had been adequately provided with colostrum, and the growth state had been good. Moreover, in the present case, pathologic changes in the umbilical artery and hind leg were completely unilateral, and the septic symptoms were not recognized after birth. In the histopathological examination, systemic purulent inflammation associated with septicemia was not observed. Therefore, the arthritis seems not to have been caused by septicemia.

In the hind limb of the horse, the umbilical artery diverges from the internal pudendal artery [3]. After birth, the umbilical artery becomes a round ligament of the bladder, and presents in rudimentary form from the bladder to the umbilical region [5]. In the present case, the thrombus in the umbilical artery spread to the point of divergence from the internal pudendal artery, suggesting that ascending infection occurred from the navel to the internal pudendal artery through the umbilical artery. Subsequently, the infection may have spread through the bloodstream, and formed abscesses on the buttocks.

It is reported that abscess and cellulitis in the soft tissues around the joint often cause arthritis of the hip and femorotibial joints [2]. In the present case, we consider the suppurative inflammation infiltrated the connective tissue of the fascias of the gluteal muscle, and spread to hip joint. Subsequently, osteomyelitis may have occurred in the left hip joint, followed by necrosis of the hip bone and femoral head. Osteonecrosis and osteolysis would have caused the dislocation of the hip joint. In the present case, there were abscesses in wide areas of the left lower thigh, and a white thrombus was formed in the left femoral vein. Therefore, it is possible that the inflammation of the buttocks may have spread to the thigh and lower thigh through blood circulation, and as a result, abscesses were formed broadly. In a bacteriological examination, bacteria were detected only in the left lower thigh, but not in the left umbilical artery and hip joint. These results might show that the lower thigh was the latest infection focus, and suppurative inflammation of the umbilical artery and hip joint had already stopped at the time of pathological autopsy.

External evidence in the umbilical region was not found in the present case. If we had examined the umbilicus and suspected umbilical infection at an early stage, some kind of treatment would have been possible before the infection spread to the hip joint. In foals, even if local symptoms of the umbilicus are not seen, it is possible that umbilical cord infection is a primary infection $[1,7,8]$. In conclusion, we should consider umbilical infection as an important differential diagnosis in inflammatory diseases of foals. In addition, it is essential to sterilize the umbilical cord thoroughly, and feed colostrums appropriately after birth.

\section{Acknowledgements}

A part of this study was supported by Tokachi A. M. A. A. and Obihiro University of Agriculture and Veterinary Medicine. 


\section{References}

1. Adams, S.B., and Fessler, J.F. 1987. Umbilical cord remnant infections in foals: 16 cases (1975-1985). J. Am. Vet. Med. Assoc. 190: 316-318.

2. Knottenbelt, D.C. 2004. Neonatal syndromes. pp. 197-182, 316-319,330-333. In: Equine Neonatology, Saunders, London.

3. Konig, H.E. 2009. Organs of the cardiovascular system. pp. 466-467. In: Veterinary Anatomy of Domestic Mammals, 4th ed., Schattaner, New York.

4. Reed, S.M. 2004. Disorders of foals. pp. 14021403. In: Equine Internal Medicine, Saunders, Philadelphia.

5. Reef, V.B., and Collatos, C. 1988. Ultrasonography of umbilical structures in clinically normal foals. Am. J. Vet. Res. 49: 2143-2146.

6. Reef, V.B., Collatos, C., Spencer, P.A., Orsini, J.A., and Sepesy, L.M. 1989. Clinical, ultrasonographic, and surgical findings in foal with umbilical remnant infections. J. Am. Vet. Med. Assoc. 195: 6972.

7. Smith, B.P. 2009. Neonatal infection. pp. 281-282. In: Large Animal Internal Medicine, Mosby Elsevier, St. Louis.

8. Staller, G.S., Tulleners, E.P., Reef, V.B., and Spencer, P.A. 1995. Concordance of ultrasonographic and physical findings in cattle with an umbilical mass or suspected to have infection of the umbilical cord remnants: 32 cases (1987-1989). J. Am. Vet. Med. Assoc. 206: 77-82. 